\title{
MEMBERS OF THE SOCIETY.
}

January 1, 1915

[Life members are designated by *]

Abbe, Prof. Cleveland. U. S. Weather Bureau, Washington, D. C. Ackermann-Teubner, Dr. B. G. A. B. G. Teubner, Leipzig, Germany. ADAMS, Prof. E. P. Princeton University, Princeton, N. J.

ADKINs, L. K. Instructor, University of Minnesota, Minneapolis, Minn. 126 Folwell Hall.

AGARD, Dr. H. L. Instructor, Williams College, Williamstown, Mass.

Akers, Prof. O. P. Alleghany College, Meadville, Pa.

ALEXANDER, J. W., II. Instructor, Princeton University, Princeton, N. J. 19 Madison Stręet.

Allardice, Prof. R. E. Stanford University, Cal.

AlleN, Dr. E. S. Instructor, Brown University, Providence, R. I.

Allen, Fiske. Supervisor, State Normal School, Charleston, Ill.

Allen, Dr. Florence E. Instructor, University of Wisconsin, Madison, Wis. 219 Lathrop Street.

ALLen, Asst. Prof. Joseph. College of the City of New York, New York, N. Y.

Allen, Prof. R. B. Kenyon College, Gambier, Ohio.

ALTSHILlER, Dr. NATHAN. Instructor, University of Washington, Seattle, Wash.

Ames, Asso. Prof. L. D. University of Missouri, Columbia, Mo. 208 Thilly Avenue.

Ammerman, Charles. McKinley Manual Training High School, St. Louis, Mo.

ANdereGG, Prof. Frederick. Oberlin College, Oberlin, Ohio. 207 East College Street.

ANDERSON, W. E. Instructor, University of Minnesota, Minneapolis, Minn.

ANDREws, Dr. Grace. 47 Brevoort Place, Brooklyn, N. Y.

*Archibald, Asst. Prof. R. C. Brown University, Providence, R. I. 9 Charles Field Street.

Armstrong, Prof. G. N. Ohio Wesleyan University, Delaware, Ohio.

Ashcraft, Prof. T. B. Colby College, Waterville, Me. 34 Pleasant Street.

Ashton, Asso. Prof. C. H. University of Kansas, Lawrence. Kan. 1200 Ohio Street. 
*Atchison, Prof. C. S. Washington and Jefferson College, Washington, Pa. 102 South Wade Avenue.

Babb, Asst. Prof. M. J. University of Pennsylvania, Philadelphia, Pa.

Bacon, Prof. Clara L. Goucher College, Baltimore, Md. 2316 North Calvert Street.

BaIlex, Prof. F. H. Massachusetts Institute of Technology, Boston, Mass. 491 Boylston Street.

BAKER, Prof. ALFRED. University of Toronto, Toronto, Canada.

BAKER, Asst. Prof. R. P. Iowa State University, Iowa City, Ia.

BARNEx, Dr. IDA. Instructor, Smith College, Northampton, Mass. 8 Paradise Road.

Barnum, Dr. Charlotte C. 344 Humphrey Street, New Haven, Conn.

BARRow, Dr. D. F. Instructor, University of Texas, Austin, Tex.

Bartlett, Prof. D. P. Massachusetts Institute of Technology, Boston, Mass.

Barton, Prof. R. M. Lombard College, Galesburg, Ill.

Barton, Prof. S. M. University of the South, Sewanee, Tenn.

Bateman, Dr. Harry. Lake and Bellona Avenues, Govans, Md.

Bates, Asso. Prof. W. H. Purdue University, Lafayette, Ind.

BaUer, Prof. G. N. University of Minnesota, Minneapolis, Minn.

BeAL, Dr. F. W. Instructor, University of Pennsylvania, Philadelphia, Pa. College Hall.

Beal, Dr. W. O. Assistant Astronomer, University of Minnesota, Minneapolis, Minn. The Observatory.

Beatty, SAmuel. Lecturer, University of Toronto, Toronto, Canada.

BECKETT, C. H. Actuary, State Life Insurance Company, Indianapolis, Ind.

BeErce, Dr. R. D. Instructor, Dartmouth College, Hanover, N. H. Austin Avenue.

Beman, Prof. W. W. University of Michigan, Ann Arbor, Mich. 813 East Kingsley Street.

Benedict, Prof. H. Y. University of Texas, Austin, Tex.

Benedict, Asso. Prof. Susan R. Smith College, Northampton, Mass.

Bennetr, A. A. Instructor, Princeton University, Princeton, N. J. 19 Madison Street.

Bernstein, Dr. B. A. Instructor, University of California, Berkeley, Cal. 2131 Haste Street.

*Berry, Arthur. Fellow and Assistant Tutor, King's College, Cambridge, England.

Berry, Asst. Prof. W. J. Polytechnic Institute of Brooklyn, Brooklyn, N. Y. 224 St. John's Place.

Betz, William. Vice-Principal, East High School, Rochester, N. Y. 160 Grand Avenue.

BIKLE, C. E. Central High School, Syracuse, N. Y. 
Bill, Asst. Prof. E. G. Dartmouth College, Hanover, N. H.

Birkhoff, Asst. Prof. G. D. Harvard University, Cambridge, Mass. 44 Shepard Street.

Blichfeldt, Asso. Prof. H. F. Stanford University, Cal.

*Burss, Prof. G. A. University of Chicago, Chicago, Ill.

Blumberg, Asst. Prof. Henry. University of Nebraska, Lincoln, Neb.

*Bôcher, Prof. Maxime. Harvard University, Cambridge, Mass. 48 Buckingham street.

Bolza, Prof. Oskar. University of Freiburg, Freiburg i. B., Germany. Reichsgrafenstrasse 10.

Börger, Dr. R. L. Associate, University of Illinois, Urbana, Ill. 1105 West Oregon Street.

Boulad, Farid. Engineer, Bridges Department, State Railways, Cairo, Egypt.

*Bouton, Asst. Prof. C. L. Harvard University, Cambridge, Mass. 5 Avon Street.

Boutroux, Prof. Ptarke. Princeton University, Princeton, N. J. Graduate College.

Bowden, Prof. JosepH. Adelphi College, Brooklyn, N. Y. 24 Clifton Place.

Boyd, Prof. P. P. University of Kentucky, Lexington, Ky.

Bracketr, Prof. F. P. Pomona College, Claremont, Cal.

Bradshaw, Asst. Prof. J. W. University of Michigan, Ann Arbor, Mich. 7\%0 Church Street.

Brand, Asst. Prof. LouIs. University of Cincinnati, Cincinnati, Ohio.

Bratton, Prof. W. A. Whitman College, Walla Walla, Wash. 570 Boyer Avenue.

BreckenRIdGe, W. E. Lecturer, Teachers College, Columbia University, New York, N. Y. 345 East 15th Street.

Brenke, Prof. W. C. University of Nebraska, Lincoln, Neb. 1250 South 21st Street.

BRewster, J. A. Instructor, College of the City of New York, New York, N. Y. 728 West 181st Street.

Bromwich, Dr. T. J. I'A. Lecturer, Cambridge University, Cambridge, England. 1 Selwyn Gardens.

Brooke, Prof. W. E. University of Minnesota, Minneapolis, Minn.

Brooks, Asst. Prof. C. E. University of California, Berkeley, Cal.

Brower, G. G. State Model School, Trenton, N. J.

*Brown, Prof. E. W. Yale University, New Haven, Conn. 116 Everit Street.

Brown, Prof. G. L. South Dakota State College, Brookings, S. D.

*Brown, J. S. Consulting Engineer, Far View, P. O. Box 38, Black Hall, Conn.

Brown, Dr. T. H. Instructor, Sheffield Scientific School, New Haven, Conn. 
Buchanan, Asst. Prof. Danier. Queen's University, Kingston, Ontario, Canada. 142 Stuart Street.

Buchanan, Prof. H. E. University of Tennessee, Knoxville, Tenn.

BucK, Dr. Thomas. Instructor, University of California, Berkeley, Cal. Faculty Club.

*Bullard, Prof. W. G. Syracuse University, Syracuse, N. Y. 124 Redfield Place.

Bullis, A. R. Macedon, Wayne Co., N. Y.

Burgess, Asst. Prof. H. T. University of Wisconsin, Madison, Wis. 812 West Dayton Street.

BuRgess, Dr. R. W. Instructor, Cornell University, Ithaca, N. Y. 302 Wait Avenue.

BURNS, Dr. JoSEPHINE E. Instructor, University of Illinois, Urbana, IIl. 920 West Illinois Street.

Bussey, Asso. Prof. W. H. University of Minnesota, Minneapolis, Minn.

Butrs, Jun. Prof. W. H. University of Michigan, Ann Arbor, Mich. 919 Oakland Avenue.

Byerly, Prof. W. E. Harvard University, Cambridge, Mass. 39 Hammond Street.

CaIn, Prof. William. University of North Carolina, Chapel Hill, N. C.

CaIrns, Asso. Prof. W. DeW. Oberlin College, Oberlin, Ohio. 55 East Lorain Street.

CaJori, Prof. Florian. Colorado College, Colorado Springs, Colo. 1119 Wood Avenue.

*Camp, Prof. B. H. Wesleyan University, Middletown, Conn. 41 Brainerd Avenue.

Campbell, Prof. D. F. Armour Institute of Technology, Chicago, Ill.

CAMPBell, Dr. G. A. Research Engineer, American Telephone and Telegraph Company, New York, N. Y. 15 Dey Street.

Caparo, Prof. J. A. Notre Dame University, Notre Dame, Ind. P. $O$. Box 54.

CAPron, PaUl. Instructor, U. S. Naval Academy, Annapolis, Md. 179 Duke of Gloucester Street.

CAReY, Asst. Prof. E. F. A. University of Montana, Missoula, Mont.

Carmichael, Asso. Prof. R. D. Indiana University, Bloomington, Ind. 521 South Grant Street.

CARPENTER, Prof. A. F. University of Washington, Seattle, Wash. 582\% Drexel Avenue, Chicago, Ill.

Carruth, Asso. Prof. W. M. Hamilton College, Clinton, N. Y. P. O. Box 25.

Carter, Asst. Prof. B. E. Colby College, Waterville, Me.

CARver, Asst. Prof. W. B. Cornell University, Ithaca, N. Y. 201 Fairmount Avenue. 
Castle, E. W. 16 East Witherspoon Hall, Princeton, N. J.

Chace, Dr. A. B. Chancellor, Brown University, Providence, R. I. 324 Angell Street.

Chambers, Asst. Prof. G. G. University of Pennsylvania, Philadelphia, Pa. 79 Drexel Avenue, Lansdowne, $P a$.

Chapman, Prof. F. E. Southern University, Greensboro, Ala.

ChITTENDEN, Dr. E. W. Instructor, University of Illinois, Champaign, Ill. 1116 Arbor Court.

Chittenden, Prof. J. B. Brooklyn Polytechnic Institute, Brooklyn, N. Y.

Clark, Prof. J. E. 34 South Park Terrace, Longmeadow, Mass.

Clarke, E. H. Instructor, Purdue University, West Lafayette, Ind. 418 Vine Street.

Clements, Dr. G. R. Instructor, University of Wisconsin, Madison, Wis. 310 North Murray Street.

Clevenger, C. H. 831 Laramie Street, Manhattan, Kan.

CoAR, Prof. H. L. Marietta College, Marietta, Ohio. Muskingum Terrace.

Совв, Asso. Prof. C. W. Amherst College, Amherst, Mass.

Совв, Prof. H. E. Lewis Institute, Chicago, Ill.

Coble, Asso. Prof. A. B. Johns Hopkins University, Baltimore, Md.

Codpington, Dr. Emily. 24 West 58th Street, New York, N. Y.

Cofrin, Asst. Prof. J. G. College of the City of New York, New York, N. Y.

Cohen, Dr. Abraham. Associate, Johns Hopkins University, Baltimore, Md.

Colaw, J. M. P. O. Box 106, Monterey, Va.

*Cole, Prof. F. N. Columbia University, New York, N. Y.

Collins, Dr. J. V. State Normal School, Stevens Point, Wis. 217 Division Street.

ColpitTs, Asst. Prof. E. C. State College of Washington, Pullman, Wash. 410 Montgomery Street.

Colpitis, Asst. Prof. Julia T. Iowa State College, Ames, Iowa.

Comstock, Prof. C. E. Bradley Polytechnic Institute, Peoria, Ill.

Conant, Prof. L. L. Polytechnic Institute, Worcester, Mass. 254 Salisbury Street.

Conner, Dr. J. R. Associate, Bryn Mawr College, Bryn Mawr, Pa.

CoNwell, Dr. G. M. Instructor, Yale University, New Haven, Conn. Box 681, Yale Station.

*Coolidge, Asst. Prof. J. L. Harvard University, Cambridge, Mass. 7 Fayerweather Street.

Copetand, Dr. LenNie P. Instructor, Wellesley College, Wellesley, Mass. 86 Shafer Hall.

Corex, S. A. Hiteman, Iowa.

Cornish, W. A. State Normal School, Cortland, N. Y. 32 Owego Street. 
Cowley, Asst. Prof. Elizabeth B. Vassar College, Poughkeepsie, N. Y. Cox, C. S. Principal, Mulberry Senior High School, Mulberry, Fla.

CRAIG, Dr. C. F. Instructor, Cornell University, Ithaca, N. Y. 417 North Aurora Street.

CrambleT, Dr. W. H. Instructor, University of Rochester, Rochester, N. Y.

Crathorne, Dr. A. R. Associate, University of Illinois, Champaign, Ill. 1113 South Fourth Street.

Crawley, Prof. E. S. University of Pennsylvania, Philadelphia, Pa.

Cresse, Asst. Prof. G. H. Middlebury College, Middlebury, Vt. 38 South Street.

Cromwell, J. W., JR. M Street High School, Washington, D. C. 1815 Thirteenth Street, $N . W$.

Cummings, Dr. Louise D. Instructor, Vassar College, Poughkeepsie, N. Y.

${ }^{*}$ Cunningham, Lieut. Col. A. J. C. 20 Essex Villas, Kensington, London, W., England.

Cunningham, Prof. Susan J. 107 North 34th Street, Philadelphia, Pa.

CuRJEL, H. W. Actuary, La Mexicana Compañia de Seguros sobre la Vida, Mexico, D. F., Mexico. Apartado 651.

CURRIER, C. H. Instructor, Brown University, Providence, R. I.

Curis, A. M. State Normal School, Oneonta, N. Y.

Curtis, Dr. H. B. Instructor, Columbia University, New York, N. Y. 401 West 118th street.

CuRTiss, Prof. D. R. Northwestern University, Evanston, Ill. 720 Milburn Street.

DaLAKER, Asst. Prof. H. H. University of Minnesota, Minneapolis, Minn. 523 Walnut Street, S. E.

Daniell, Prof. P. J. Rice Institute, Houston, Texas.

Daniels, Dr. A. L., Jr. Instructor, Yale University, New Haven, Conn. 102 York Square.

Davis, Mrs. E. B. Nautical Almanac Office, Washington, D. C. 2212 First Street, N. W.

Davis, Prof. E. W. University of Nebraska, Lincoln, Neb.

*Davis, Asst. Prof. H. N. Harvard University, Cambridge, Mass. 8 Ash Street Place.

Davis, Asso. Prof. J. M. University of Kentucky, Lexington, Ky. 340 Madison Place.

Davis, Prof. N. F. Brown University, Providence, R. I. 159 Brown Street.

Davisson, Prof. S. C. Indiana University, Bloomington, Ind. 515 East Third Street.

DeCHeRd, Miss M. E. Instructor, University of Texas, Austin, Tex. 2313 Nueces Street. 
Decker, Asso. Prof. F. F. Syracuse University, Syracuse, N. Y. 854 Sumner Avenue.

DE Cou, Prof. E. E. University of Oregon, Eugene, Ore. 1135 Mill Street.

*DeDerICK, Dr. L. S. Instructor, Princeton University, Princeton, N. J. P. O. Box 105.

Defoe, Prof. L. M. University of Missouri, Columbia, Mo.

Deimer, Asst. Prof. R. F. Stevens Institute of Technology, Hoboken, N. J.

DeLong, Prof. I. M. University of Colorado, Boulder, Colo. 1841 Broadway.

DeLury, Prof. A. T. University of Toronto, Toronto, Canada.

*Dennetr, Dr. W. S. 8 East 49th Street, New York, N. Y.

Denton, Dr. W. W. Instructor, University of Illinois, Champaign, Ill. 905 South Sixth Street.

Dickson, Prof. L. E. University of Chicago, Chicago, Ill. 5535 University Avenue.

DimicK, C. E. Instructor, Revenue Cutter Academy, New London, Conn. 41 Squire Street.

DINES, C. R. Instructor, Northwestern University, Evanston, Ill. 1925 Sherman Avenue.

Dines, Asst. Prof. L. L. University of Saskatchewan, Saskatoon, Canada.

Dodd, Adj. Prof. E. L. University of Texas, Austin, Tex.

DoHmen, Dr. F. J. Instructor, Harvard University, Cambridge, Mass. 21 Walker Street.

Dowling, Asso. Prof. L. W. University of Wisconsin, Madison, Wis. 2 Roby Road.

DRESDEN, Asst. Prof. ARNOLD. University of Wisconsin, Madison, Wis. 2114 Vilas Street.

Dunkel, Dr. Отто. Instructor, University of Missouri, Columbia, Mo. 602 Sanford Street.

DuRELl, Dr. Fletcher. Lawrenceville School, Lawrenceville, N. J.

Durfee, Prof. W. P. Hobart College, Geneva, N. Y.

Duval, Asso. Prof. E. P. R. University of Oklahoma, Norman, Okla. 629 Boyd Street.

Echols, Prof. C. P. U. S. Military Academy, West Point, N. Y. Echols, Prof. W. H. University of Virginia, Charlottesville, Va.

EDDy, Prof. H. T. University of Minnesota, Minneapolis, Minn. 916 Sixth Street, S. $E$.

Edmondson, Prof. T. W. New York University, University Heights, New York, N. Y.

Edwards, Prof. G. C. University of California, Berkeley, Cal. 2546 Dana Street.

Eells, W. C. Instructor, U. S. Naval Academy, Annapolis, Md. 
EIEsLand, Prof. JoHn. University of West Virginia, Morgantown, W. Va.

*Eisenhart, Prof. L. P. Princeton University, Princeton, N. J. 22 Alexander Street.

*Elliotr, Prof. E. B. Oxford University, Oxford, England. 4 Bardwell Road.

EMCH, Asst. Prof. ARNoLd. University of Illinois, Urbana, Ill. 604 West Elm Street.

Emmons, Prof. C. W. Simpson College, Indianola, Iowa.

ENGBerg, Prof. C. C. University of Nebraska, Lineoln, Neb. 330 North 32d Street.

Engler, Dr. E. A. Secretary and Treasurer, Washington University, St. Louis, Mo.

ENGLISH, HarRY. Head of the Mathematical Department, Washington High Schools, Washington, D. C. 2907 P Street, N.W.

Epsteen, Dr. Saul. State Insurance Commissioner, Denver, Colo. State Capitol.

*Escotr, E. B. Actuary, Peninsular Insurance Company, Detroit, Mich. 627 Majestic Building.

Estr, Prof. T. C. Amherst College, Amherst, Mass.

Esty, Prof. W. C. Amherst College, Amherst, Mass. 85 Elm Street, Worcester, Mass.

Entringer, H. J. Instructor, University of Texas, Austin, Tex.

*Evans, Asst. Prof. G. C. Rice Institute, Houston, Tex.

Evans, G. W. Charlestown High School, Boston, Mass.

Evans, Prof. H. B. University of Pennsylvania, Philadelphia, Pa. College Hall.

Faufhe, Prof. J. B. Kent, Ohio.

FeEmster, Prof. H. C. York College, York, Neb.

*Ferry, Prof. F. C. Williams College, Williamstown, Mass.

Field, Prof. Floxd. Georgia School of Technology, Atlanta, Ga. 91 Bryan Street.

Field, Jun. Prof. Peter. University of Michigan, Ann Arbor, Mich. 1054 Ferdon Road.

*Fields, Prof. J. C. University of Toronto, Toronto, Canada.

Findlay, Prof. William. McMaster University, Toronto, Canada.

Fine, Prof. H. B. Princeton University, Princeton, N. J.

Finkel, Prof. B. F. Drury College, Springfield, Mo.

Fischer, Dr. C. A. Instructor, Columbia University, New York, N. Y.

Fisher, Prof. G. E. University of Pennsylvania, Philadelphia, Pa. The College.

Fisher, Prof. Irving. Yale University, New Haven, Conn. 460 Prospect Street. 
*Fiske, Prof. T. S. Columbia University, New York, N. Y. Fitch, Mrs. EDWARd. Clinton, N. Y.

Frte, Prof. W. B. Columbia University, New York, N. Y.

FITTERER, Prof. J. C. University of Wyoming, Laramie, Wyo. 511 South 11th Street.

Flanagan, C. E. Actuary, Conservative Life Insurance Company, Wheeling, W. Va.

Fleet, Prof. R. R. William Jewell College, Liberty, Mo.

Focke, Prof. T. M. Case School of Applied Science, Cleveland, Ohio.

Ford, L. R. Lecturer, University of Edinburgh, Edinburgh, Scotland. 103 Newington Road.

*Ford, Jun. Prof. W. B. University of Michigan, Ann Arbor, Mich, 904 Forest Avenue.

Forsyth, Prof. A. R. Imperial College of Science and Technology, London, S. W., England.

Fort, Dr. Tomlinson. Instructor, University of Michigan, Ann Arbor, Mich. 819 South state Street.

*Frankland, F. W. Government Examiner, Foxton, New Zealand. Okataina.

Franklin, Dr. Fabian. The Evening Post, New York, N. Y.

Franklin, Prof. W. S. Lehigh University, South Bethlehem, Pa.

Frazer, F. D. 1206 Haight Avenue, Portland, Ore.

Frizeld, Prof. A. B. McPherson College, McPherson, Kan. 1010 East Euclid Street.

GABA, Dr. M. G. Instructor, Carnegie Institute of Technology, Pittsburgh, $\mathrm{Pa}$

GALE, Prof. A. S. University of Rochester, Rochester, N. Y.

GARRETSON, W. VAN N. Instructor, University of Michigan, Ann Arbor, Mich. 1345 Wilmot Street.

GARRETt, Prof. W. H. Baker University, Baldwin, Kan. 230 Upland Road, Cambridge, Mass.

GarRIson, Asst. Prof. W. A. Union University, Schenectady, N. Y. 2 Rosa Road.

Gavetr, Asst. Prof. G. I. University of Washington, Seattle, Wash. 5608 Washington Boulevard.

Gentri, Dr. Ruth. Stilesville, Ind.

Germann, Dr. G. B. Principal of Public School No. 130, Brooklyn, N.

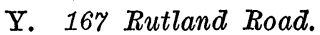

*Gerrans, H. T. Fellow and Lecturer, Worcester College, Oxford, England. 20 St. John Street.

Gibson, Prof. G. A. University of Glasgow, Glasgow, Scotland. 10 The University.

Gillespie, Asst. Prof. D. C. Cornell University, Ithaca, N. Y. 214 University Avenue. 
Gillespie, Prof. William. Princeton University, Princeton, N. J. 253 Nassau Street.

GLashan, Dr. J. S. C. Ottawa, Canada.

Glazier, Prof. Harriet E. The Western College, Oxford, Ohio.

Glenn, Prof. O. E. University of Pennsylvania, Philadelphia, Pa. College Hall.

GLover, Prof. J. W. University of Michigan, Ann Arbor, Mich. 620 Oxford Road.

GoRe, J. K. Vice-President and Actuary, Prudential Insurance Com. pany, Newark, N. J.

GottschaLI, LouIs. Star Ribbon Manufacturing Company, 462 Broadway, New York, N. Y.

GRABER, Prof. M. E. Heidelberg University, Tiffin, Ohio, 122 Circular Street.

Graham, W. J. Actuary, Equitable Life Assurance Society, 165 Broadway, New York, N. Y.

Grant, Asst. Prof. E. D. Michigan College of Mines, Houghton, Mich. 124 Hubbell Aivenue.

Granville, Pres. W. A. Pennsylvania College, Gettysburg, Pa.

Graustein, Dr. W. C. Instructor, Rice Institute, Houston, Tex.

Gravatt, T. E. Instructor, Pennsylvania State College, State College, $\mathrm{Pa}$.

Graves, Dr. G. H. Instructor, Columbia University, New York, N. Y. Hamilton Hall.

GREEN, Dr. G. M. Instructor, Harvard University, Cambridge, Mass. 27 Walker Street.

*GREenhild, Sir George. 1 Staple Inn, London, W. C.

Grennan, Dr. Elizabeth B. Instructor, University of Nebraska, Lincoln, Neb. Station $A$.

GRIFrin, Prof. F. L. Reed College, Portland, Ore.

GRIFFith, J. H. Associate Engineer-Physicist, U. S. Bureau of Standards, Pittsburgh, Pa. 39th and Butler Streets.

GrImes, Mr. N. C. Instructor, University of Illinois, Urbana, Ill. Room 42\%, New Hampshire Building.

*GRoAT, B. F. Engineer. 2400 Oliver Building, Pittsburgh, Pa.

Gronwall, Asst. Prof. T. H. Princeton University, Princeton, N. J. 12 Nassau Street.

Grove, Asst. Prof. C. C. Columbia University, New York, N. Y. Hamilton Hall.

Gummer, Asst. Prof. C. F. Queen's University, Kingston, Ontario, Canada. 137 Union Street.

*Gummere, Prof. H. V. Drexel Institute, Philadelphia, Pa.

Gundersen, Prof. CARL. Oklahoma Agricultural and Mechanical College, Stillwater, Okla. 217 College Avenue.

Gunther, Prof. C. O. Stevens Institute of Technology, Hoboken, N. J. P. O. Box $7 \%$. 
HALL, Prof. A. G. University of Michigan, Ann Arbor, Mich. 1036 Oakland Avenue.

Hallett, Prof. G. H. University of Pennsylvania, Philadelphia, Pa. College Hall.

*Halsted, Prof. G. B. 942 Ninth Avenue, Greeley, Colo.

*Hanawalt, Prof. F. W. University of Puget Sound, Tacoma, Wash. 826 North Steele Street.

HaNCOCK, Prof. Harris. University of Cincinnati, Cincinnati, Ohio.

HanNa, Asso. Prof. U. S. Indiana University, Bloomington, Ind.

*Hardcastle, Miss Frances. 3 Osborne Terrace, Newcastle-on-Tyne, England.

Hardy, Prof. J. G. Williams College, Williamstown, Mass.

*Harkness, Prof. James. McGill University, Montreal, Canada.

Harshbarger, Prof. W. A. Washburn College, Topeka, Kan. 1401 College Avenue.

HART, Prof. J. N. University of Maine, Orono, Me.

HART, Prof. W. W. University of Wisconsin, Madison, Wis.

Hartwell, Prof. G. W. Hamline University, St. Paul, Minn. 7 y $^{7}$ Fry Street.

Harvey, Prof. H. C. Armstrong, Mo.

Haseman, Prof. Charles. University of Nevada, Reno, Nev.

Haskeld, Prof. M. W. University of California, Berkeley, Cal. P. $O$. Box 3.

Haskins, Asst. Prof. C. N. Dartmouth College, Hanover, N. H. $R . \boldsymbol{F}$. D. 1, Lebanon, N. H.

Hathaway, Prof. A. S. Rose Polytechnic Institute, Terre Haute, Ind.

Hawkes, Prof. H. E. Columbia University, New York, N. Y.

Hawkesworth, Rev. A. S. Sheridanville, Pittsburgh, Pa.

HAYASHI, Prof. Tsuruichi. College of Science, Tohoku Imperial University, Sendai, Japan.

Hayes, G. M. Instructor, College of the City of New York, New York, N. Y. 3091 Decatur Avenue.

H'Doubler, Dr. F. T. 227 Aspinwall Avenue, Brookline, Mass.

HEDRICK, Prof. E. R. University of Missouri, Columbia, Mo.

Henderson, Robert. Actuary, Equitable Life Assurance Society, 165 Broadway, New York, N. Y.

Hennel, Dr. CoRA B. Instructor, Indiana University, Bloomington, Ind. 822 East Third Street.

Hewes, Dr. L. I. Office of Public Roads, Washington, D. C.

Higdon, J. E. Actuary, State Department of Insurance, Austin, Tex. State Capitol.

Higlex, Asst. Prof. H. R. Stevens Institute, Hoboken, N. J.

HILDEBRANDT, Dr. T. H. Instructor, University of Michigan, Ann Arbor, Mich. 513 Elm Street. 
Hiltebeitel, Dr. A. M. Trappe, Montgomery Co., Pa.

Himowich, Dr. A. A. 1913 Madison Avenue, New York, N. Y.

HiтснсосK, Asso. Prof. R. R. University of North Dakota, University, N. Dak.

Hodae, Prof. F. H. Franklin College, Franklin, Ind. 670 Jefferson Street.

Hodge, Prof. Percy. Stevens Institute of Technology, Hoboken, N. J. 99 Claremont Avenue, New York, $N . \bar{Y}$.

Hodgkins, Prof. H. L. George Washington University, Washington, D. C.

Hogrefe, L. T. W. Chemist. 1119 Park Place, Brooklyn, N. Y.

Holgate, Prof. T. F. Northwestern University, Evanston, Ill. 617 Library Street.

Hopkins, L. A. Instructor, University of Michigan, Ann Arbor, Mich. 1203 Church Street.

Hoskins, Prof. L. M. Stanford University, Palo Alto, Cal. 365 Lincoln Avenue.

Howe, Pres. C. S. Case School of Applied Science, Cleveland, Ohio.

Howe, Dr. H. A. Astronomer. University Park, Colo.

Howland, Asso. Prof. L. A. Wesleyan University, Middletown, Conn. 34 Home Avenue.

Hulburt, Prof, L. S. Johns Hopkins University, Baltimore, Md.

Hun, Asst. Prof. J. G. Princeton University, Princeton, N. J.

Huntington, Asst: Prof. E. V. Harvard University, Cambridge, Mass. 27 Everett Street.

Hurwitz, Asst. Prof. W. A. Cornell University, Ithaca, N. Y. 8 White Hall.

Hussex, Prof. W. J. University of Michigan, Ann Arbor, Mich.

Hutchinson, Prof. J. I. Cornell University, Ithaca, N. Y. 30 Thurston Avenue.

Hyde, E. W. Actuary, Columbia Life Insurance Company, Cincinnati, Ohio. 814 Lincoln Avenue, Station D.

INGoLd, Asst. Prof. Louis. University of Missouri, Columbia, Mo. 206 Thilly Avenue.

IRwIN, Dr. Frank. Instructor, University of California, Berkeley, Cal. 2632 Haste Street.

JACKson, Dr. Dunham. Instructor, Harvard University, Cambridge, Mass. 5 Conant Hall.

JACKson, Dr. L. L. Principal, Baldwin Street School, Montclair, N. J. $1^{\text {t7 }}$ Oxford Street.

JAcoBus, D. S. Advisory Engineer, Babcock and Wilcox Company, 85 Liberty Street, New York, N. Y.

James, Asst. Prof. G. O. Washington University, St. Louis, Mo. 
JoFfe, S. A. Assistant Actuary, Mutual Life Insurance Company, New York, N. Y. 55 Cedar Street.

Johnson, Prof. B. F. Missouri State Normal School, Cape Girardeau, Mo.

Johnson, Dr. R. A. Instructor, Adelbert College, Western Reserve University, Cleveland, Ohio. 1489 East 120th Street.

Johnson, Prof. W. W. U. S. Naval Academy, Annapolis, Md. 909 St. Paul Street, Baltimore, $\mathbf{M} d$.

Jones, Dr. C. C. Chancellor, University of New Brunswick, Fredericton, N. B., Canada.

Jones, Prof. E. H. Daniel Baker College, Brownwood, Tex. 601 Coggin Avenue.

Jones, Dr. J. L. Instructor, University of Pittsburgh, Pittsburgh, Pa.

JoRDAN, Asst. Prof. H. E. University of Kansas, Lawrence, Kan. 1600 Kentucky Street.

KarpinsKI, Asst. Prof. L. C. University of Michigan, Ann Arbor, Mich. 1315 Cambridge Road.

*Kasner, Prof. Edward. Columbia University, New York, N. Y. 22 West 119th Street.

KELLOGG, Prof. O. D. University of Missouri, Columbia, Mo. 307 Thilly Avenue.

KeLLS, Dr. L. M. 502 West 122d Street, New York, N. Y.

KEMPNER, Dr. A. J. Instructor, University of Illinois, Urbana, Ill.

Kenneliz, Prof. A. E. Harvard University, Cambridge, Mass.

Kent, Asso. Prof. F. C. University of Oklahoma, Norman, Okla. 524 Webster Avenue.

*Kenyon, Prof. A. M. Purdue University, Lafayette, Ind.

KEPPEL, Prof. H. G. University of Florida, Gainesville, Fla.

*Keyser, Prof. C. J. Columbia University, New York, N. Y.

KILLAM, Dr. S. D. Lecturer, University of Alberta, Edmonton, Canada.

KINDLE, J. H. Instructor, University of Cincinnati, Cincinnati, Ohio.

KInGSTON, Dr. H. R. Lecturer, University of Manitoba, Winnipeg, Canada,

KTRCHER, Dr. E. A. T. Instructor, Massachusetts Institute of Technology, Boston, Mass.

KNisely, AleXander. Columbia City, Ind.

KocH, E. H., Jr. High School of Commerce, New York, N. Y. 874 South 15th Street, Newark, N. J.

KorN, Prof. ARTHUR. Charlottenburg Technical School, Charlottenburg, Germany. Schlüterstrasse 25.

KrathWOHL, Asst. Prof. W. C. Armour Institute of Technology, Chicago, Ill.

Kuнn, Prof. H. W. Ohio State University, Columbus, Ohio. 1460 Michigan Avenue. 
KUSCHKE, Dr. C. G. P. Instructor, University of Washington, Seattle, Wash. 3760 14th Avenue, N. E.

KÜSTERMANN, Dr. W. W. Instructor, University of Michigan, Ann Arbor, Mich. 716 Forest Avenue.

*Ladue, Pomerox. San Francisco-Oakland Terminal Railways, Oakland, Cal.

Lambert, Prof. P. A. Lehigh University, South Bethlehem, Pa. 215 South Center Street, Bethlehem, $P$ a.

LAmbert, W. D. U. S. Coast and Geodetic Survey, Washington, D. C.

Lamond, Dr. J. K. Instructor, Wesleyan University, Middletown, Conn. 38 Brainerd Avenue.

Landis, Prof. W. W. Dickinson College, Carlisle, Pa.

LANDRY, Prof. A. E. Catholic University of America, Washington, D. C. 3624 13th Street, Brookland, D. C.

Lanza, Prof. Gaetano. The Montevista, 63d and Oxford Streets, Philadelphia, Pa.

Laves, Asso. Prof. Kurt. University of Chicago, Chicago, Ill. 5611 Kenwood Avenue.

LeFschetz, Dr. Solomon. Instructor, University of Kansas, Lawrence, Kan. 937 Missouri Street.

LeHMer, Asso. Prof. D. N. University of California, Berkeley, Cal. 2736 Regent Street.

LEIB, Dr. D. D. Instructor, Yale University, New Haven, Conn. 21 Maple Street.

Lennes, Prof. N. J. University of Montana, Missoula, Mont.

Leonard, Prof. H. B. University of Oregon, Eugene, Ore.

Lester, Prof. O. C. University of Colorado, Boulder, Colo.

Leuschner, Prof. A. O. University of California, Berkeley, Cal. Students' Observatory.

Levi-Civita, Prof. Tullio. University of Padua, Padua, Italy. Via Altinate, 14.

Lewis, Asst. Prof. Florence P. Goucher College, Baltimore, Md.

LIBBY, B. B. Port Washington, Wis.

Ligнt, G. H. Yale University, New Haven, Conn. 70 Whalley Avenue.

Linehan, P. H. Instructor, College of the City of New York, New York, N. Y. 518 West 143d Street.

Ling, Prof. G. H. University of Saskatchewan, Saskatoon, Canada.

Linton, M. A. Mathematician, Provident Life and Trust Company, 409 Chestnut Street, Philadelphia, Pa.

LIPKA, Dr. JOSEPH. Instructor, Massachusetts Institute of Technology, Boston, Mass.

LOCKE, L. L. 950 St. John's Place, Brooklyn, N. Y.

Longlex, Asst. Prof. W. R. Śheffield Scientific School, Yale University, New Haven, Conn. 266 Willow Street. 
Love, Prof. A. E. H. Oxford University, Oxford, England. 34 st. Margaret's Road.

Love, Asst. Prof. C. E. University of Michigan, Ann Arbor, Mich. 1527 South University Avenue.

*Lovetr, Pres. E. O. Rice Institute, Houston, Tex.

LuBy, W. A. Northeast High School, Kansas City, Mo.

${ }^{*}$ LudLOW, Col. H. H. Fort Stevens, Ore.

Lunn, Asst. Prof. A. C. University of Chicago, Chicago, Ill.

Lyman, Prof. E. A. Michigan State Normal College, Ypsilanti, Mich. 126 North Washington Street.

Lytle, Dr. E. B. Associate, University of Illinois, Urbana, Ill, 603 South Orchard Street.

McClenon, Asso. Prof. R. B. Grinnell College, Grinnell, Iowa. 140\% Elm Street.

*McClintock, Dr. Emory. Consulting Actuary, Mutual Life Insurance Company of New York. 2305 De Lancey Street, Philadelphia, $\mathrm{Pa}$.

McCormack, T. J. Principal, La Salle-Peru High School, La Salle, Ill.

MCDonald, Asst. Prof. J. H. University of California, Berkeley, Cal. 2337 Telegraph Avenue.

McDonneld, John. Geodetic Survey of Canada, Dominion Observatory, Ottawa, Canada.

McEwen, Dr. G. F. Oceanographer, Scripps Institution for Biological Research, La Jolla, Cal.

McKelvey, Dr. J. V. Instructor, Cornell University, Ithaca, N. Y. 3 Central Avenue.

MoKinnex, Prof. T. E. University of South Dakota, Vermillion, S. Dak. 222 North University Street.

MCMAHon, Prof. JAMES. Cornell University, Ithaca, N. Y. 7 Central Avenue.

MoNeill, Prof. Malcolm. Lake Forest College, Lake Forest, Ill.

*Macadlay, Dr. F. S. The Chesters, Vicarage Road, East Sheen, London, S. W., England.

MacDill, Lieut. LesLie. Fort Hamilton Station, Brooklyn, N. Y.

MacInnes, Asst. Prof. C. R. Princeton University, Princeton, N. J.

MacKinnon, Dr. Annie L. See Fitch, Mrs. Edward.

Maclaurin, Pres. R. C. Massachusetts Institute of Technology, Boston, Mass.

Maclay, Prof. James. Columbia University, New York, N. Y.

MacMillan, Asst. Prof. W. D. University of Chicago, Chicago, Ill. 5407 Woodlawn Avenue.

Macneill, Prof. Murray. Dalhousie University, Halifax, N. S., Canada. MACNeIsh, Dr. H. F. Instructor, Sheffield Scientific School, Yale University, New Haven, Conn. 352 Temple Street. 
Maddison, Dr. Isabel. Recording Dean, Bryn Mawr College, Bryn Mawr, Pa. Taylor Hall.

Maglott, Prof. Eva S. Ohio Northern University, Ada, Ohio.

Mahoney, J. O. High School, Dallas, Tex. 1900 Crockett Street.

Maltbie, Dr. W. H. Attorney at Law. 728 Equitable Building, Baltimore, $\mathrm{Md}$.

Manning, Asso. Prof. H. P. Brown University, Providence, R. I.

Manning, Asso. Prof. W. A. Stanford University, Palo Alto, Cal. $1698 \mathrm{D}$ Waverly Street.

MarCH, Asst. Prof. H. W. University of Wisconsin, Madison, Wis. 510 North Carroll Street.

M.ArkLex, Prof. J. L. University of Michigan, Ann Arbor, Mich. Geddes and Oxford Road.

Marshall, Asso. Prof. William. Purdue University, Lafayette, Ind. University of Arizona, Tucson, Ariz.

Martin, Dr. Artemas. U. S. Coast and Geodetic Survey, Washington, D. C. $920 \mathrm{~N}$ street, $N$. $W$.

Martin, Asso. Prof. Emilie N. Mount Holyoke College, South Hadley, Mass.

Martin, Prof. L. A. Stevens Institute of Technology, Hoboken, N. J. 911 Castle Point Terrace.

*Mason, Prof. Max. University of Wisconsin, Madison, Wis.

MASON, Dr. T. E. Instructor, Purdue University, Lafayette, Ind. 226 South Grant Street.

Mathews, R. M. Instructor, Polytechnic High School, Riverside, Cal.

MeLCher, GeORGe. Library Building, Kansas City, Mo.

Mendizábal-Tamborrel, Dr. Joaquin DE. Geographical and Military Engineer. Palma No. 13, Mexico City, Mexico.

*Merrill, Asso. Prof. Helen A. Wellesley College, Wellesley, Mass.

Merriman, Dr. Mansfield. Consulting Engineer. 1071 Madison Avenue, New York, N. Y.

*Metzler, Prof. W. H. Syracuse University, Syracuse, N. Y. 760 Comstock Avenue.

Mikesh, J. S. Instructor, Harvard University, Cambridge, Mass. 1746 Cambridge Street.

Miles, Dr. E. J. Instructor, Sheffield Scientific School, Yale University, New Haven, Conn. 115 Brownell Street.

Miller, E. A. 113 North Tioga Street, Ithaca, N. Y.

MILLER, Prof. F. E. Otterbein University, Westerville, Ohio.

*Mrller, Prof. G. A. University of Illinois, Urbana, Ill. 1103 West Illinois Street.

Miller, Prof. J. A. Swarthmore College, Swarthmore, Pa.

Miser, Dr. W. L. Instructor, College of Engineering, University of Minnesota, Minneapolis, Minn.

Mitcheld, Prof. B. E. Millsaps College, Jackson, Miss. 799 Fairview Avenue. 
Mitchell, Prof. H. B. Columbia University, New York, N. Y.

Mitchell, Asst. Prof. H. H. University of Pennsylvania, Philadelphia, Pa. College Hall.

Mitchell, Asst. Prof. U. G. University of Kansas, Lawrence, Kan. 1313 Massachusetts Street.

Moody, Prof. W. A. Bowdoin College, Brunswick, Me. 60 Federal Street.

Moore, Asst. Prof. C. L. E. Massachusetts Institute of Technology, Boston, Mass.

Moore, Asst. Prof. C. N. University of Cincinnati, Cincinnati, Ohio. 3325 Burnet Avenue.

*Moore, Prof. E. H. University of Chicago, Chicago, Ill. 5607 Kenwood Avenue.

Moone, Dr. R. L. Instructor, University of Pennsylvania, Philadelphia, Pa. 5936 Washington Avenue.

Moots, E. E. Civil Engineer. Walla Walla, Wash.

Moreno, Asso. Prof. H. C. Stanford University, Cal. Box 894.

Morgan, Dr. F. M. Instructor, Dartmouth College, Hanover, N. H.

Moritz, Prof. R. E. University of Washington, Seattle, Wash.

Morlex, Prof. Frank. Jahns Hopkins University, Baltimore, Md.

Morris. Asst. Prof. C. C. Ohio State University, Columbus, Ohio.

Morris, Prof. RICHARd. Rutgers College, New Brunswick, N. J. 94 Easton Avenue.

Morrison, Asso. Prof. F. M. University of Washington, Seattle, Wash. 4626 21st Avenue, N. $E$.

Morrow, E. B. Gilman Country School, Roland and Belvedere Avenues, Baltimore, Md.

Moulton, Asst. Prof. E. J. Northwestern University, Evanston, Ill. 909 Colfax Street.

Moulton, Asso. Prof. F. R. University of Chicago, Chicago, Ill.

Mourad, Lieut. SALIH. Third Section, First Department, Admiralty, Constantinople, Turkey.

Muir, Dr. Thomas. Education Office, Capetown, South Africa.

*MukhopâdhyÁy, Dr. Asutosh. University of Calcutta, Calcutta, India. ry Russa Road North, Bhowanipore.

Mullen, Dr. L. B. Girls' High School, Nostrand Avenue, Brooklyn, N. $Y$.

Mullins, G. W. Instructor, Columbia University, New York, N. Y.

Murray, Prof. D. A. McGill University, Montreal, Canada.

Mrens, Prof. G. W. University of Chicago, Chicago, Ill. 1953 East rod Street.

NeIKIRK, Asst. Prof. L. I. University of Washington, Seattle, Wash. 4793 21st Avenue, N. E.

Nelson, Prof. A. B. Central University, Danville, Ky. 
Newcomer, H. S. 1529 North Broadway, Baltimore, Md.

NEWKIRK, Asst. Prof. B. L. College of Engineering, University of Minnesota, Minneapolis, Minn.

Newson, Dr. Mary W. (Mrs. H. B.) 1620 Massachusetts Street, Lawrence, Kan.

Noble, Asso. Prof. C. A. University of California, Berkeley, Cal. 2224 Piedmont Avenue.

Nortнсотт, J. A. Instructor, Syracuse University, Syracuse, N. Y.

Nowlan, F. S. Columbia University, New York, N. Y. 547 West 123d Street.

NYBERG, J. A. Instructor, University of Wisconsin, Madison, Wis. 145 Iota Court.

OLDs, Prof. G. D. Amherst College, Amherst, Mass.

OsBorne, Prof. G. A. Massachusetts Institute of Technology, Boston, Mass.

OsGood, Prof. W. F. Harvard University, Cambridge, Mass. 74 Avon Hill Street.

O'Shaughnessy, Dr. Louis. Instructor, University of Pennsylvania, Philadelphia, Pa. Box 6, College Hall.

Owens, Dr. F. W. Instructor, Cornell University, Ithaca, N. Y. 110 Westburne Lane.

Paaswell, George. Civil Engineer. 2726 Creston Avenue, New York, N. Y.

PAGE, Prof. J. M. University of Virginia, Charlottesville, Va.

PaIne, Prof. G. P. University of Wisconsin, Madison, Wis.

Palmer, Asso. Prof. C. I. Armour Institute of Technology, Chicago, Ill.

Palmer, Prof. E. S. Rollins College, Winter Park, Fla.

Palmite, Prof. AnNa H. College for Women, Western Reserve University, Cleveland, Ohio. 13223 Forest Hill Avenue, East Cleveland.

*Partridge, Prof. E. A. Boys' High School, 48th and Walnut Streets, Philadelphia, Pa.

Patterson, Dr. J. L. Chestnut Hill Academy, Chestnut Hill, Philadelphia, Pa.

Patrillo, Prof. N. A. Randolph-Macon Woman's College, Lynchburg, Va.

Pedersen, Asst. Prof. F. M. College of the City of New York, New York, N. Y. 452 West 144th Street.

PeEd, Prof. M. T. Emory College, Oxford, Ga.

Pell, Dr. Alexander. South Hadley, Mass.

Pell, Asso. Prof. Anna J. (Mrs. Alexander.) Mount Holyoke College, South Hadley, Mass.

Pemberton, Prof. W. S. Wheaton College, Wheaton, Ill. 814 College Avenue. 
Pfeiffer, Dr. G. A. Tutor, College of the City of New York, New York, N. Y. 56 Anderson Avenue, Palisade, N. J.

Philip, Asst. Prof. Maximilian. College of the City of New York, New York, N. Y.

Phillips, Dr. H. B. Instructor, Massachusetts Institute of Technology, Boston, Mass.

Pierpont, Prof. James. Yale University, New Haven, Conn. 42 Mansfield Street.

Pitcher, Asst. Prof. A. D. Dartmouth College, Hanover, N. H.

Plant, Prof. L. C. Agricultural College, East Lansing, Mich.

Plimpton, Dr. G. A. Ginn and Company, 70 Fifth Avenue, New York, N. Y.

Ponzer, Asst. Prof. E. W. Stanford University, Cal.

Poor, Prof. C. L. Columbia University, New York, N. Y. 35 Thomas Street.

Poor, Asst. Prof. J. M. Dartmouth College, Hanover, N. H.

PooR, V. C. Instructor, University of Michigan, Ann Arbor, Mich. 1018 Church Street.

Porter, Prof. M. B. University of Texas, Austin, Tex.

Posex, F. D. 409 Higgins Building, Los Angeles, Cal.

Powell, H. W. Tutor, College of the City of New York, New York, N. Y. The Apthorp, 79th Street and Broadway.

Powers, R. E. Care of R. F. Watkins, Treasurer, Denver \& Rio Grande R. R. Co., Denver, Colo.

*Pupin, Prof. M. I. Columbia University, New York, N. Y.

Putnam, Asso. Prof. T. M. University of California, Berkeley, Cal.

Putnam, W. L. Attorney at Law. 60 State Street, Boston, Mass.

Ragsdale, Dr. Virginia. Jamestown, N. C.

Ranum, Asst. Prof. Arthur. Cornell University, Ithaca, N. Y. 113 Osmun Place.

RASOR, Prof. S. E. Ohio State University, Columbus, Ohio.

RAYWORTH, J. C. Instructor, Washington University, St. Louis, Mo.

Reaves, Prof. S. W. University of Oklahoma, Norman, Okla. 911 East 57th Street, Chicago, Ill.

*Reddick, Dr. H. W. Instructor, Columbia University, New York, N. Y. Livingston Hall.

ReID, Prof. L. W. Haverford College, Haverford, Pa.

Reilly, Asst. Prof. J. F. University of Iowa, Iowa City, Iowa. 624 South Governor Street.

Reilly, Dean Marion. Bryn Mawr College, Bryn Mawr, Pa.

Remick, Prof. B. L. Kansas State Agricultural College, Manhattan, Kan. 
Reynolds, C. N., Jr. 10 Conant Hall, Harvard University, Cambridge, Mass.

Reynolds, Asso. Prof. F. G. College of the City of New York, New York, N. Y.

RICE, C. M. P. O. Box 215, Worcester, Mass.

Richardson, M. R. 618 McMannen Street, Durham, N. C.

*RIChardson, Asso. Prof. R. G. D. Brown University, Providence, R. I.

Richardson, Miss S. F. Instructor, Vassar College, Poughkeepsie, N. Y.

*RICHMond, H. W. Lecturer, Cambridge University, Cambridge, England. King's College.

RIDER, P. R. Assistant, Yale University, New Haven, Conn. 16 York Square.

RIETz, Prof. H. L. University of Illinois, Urbana, Ill. 1107 West Oregon Street.

RIGGS, Prof. N. C. Carnegie Institute of Technology, Pittsburgh, Pa.

Risley, Prof. W. J. James Milliken University, Decatur, Ill. 1340 West Macon street.

Robbins, Dr. R. B. Instructor, Sheffield Scientific School, Yale University, New Haven, Conn. 169 Park Street.

Roberts, Prof. Maria M. Iowa State College, Ames, Iowa. 219 Ash Avenue.

Robinson, L. B. 306 East 22d Street, Baltimore, Md.

RoE, Prof. E. D., Jr. Syracuse University, Syracuse, N. Y. 123 West Ostrander Avenue.

Rot, Josephine R. (Mrs. E. D.) 123 West Ostrander Avenue, Syracuse, N. Y.

Rozver, Asst. Prof. W. H. Washington University, St. Louis, Mo.

Roosevelt, G. E. 30 Pine Street, New York, N. Y.

Root, Dr. R. E. Instructor, U. S. Naval Academy, Annapolis, Md. 99 Market Street.

Rorer, Dr. J. T. William Penn High School, Philadelphia, Pa. 333 North 34th Street.

Rosenbaum, Dr. Joseph. Instructor, Rosenbaum School, New Haven, Conn. 660 George Street.

Rosenblatt, Dr. AlFred. Docent, University of Krakau, Krakau, Austria. 19 Basteistrasse.

Rотнвоск, Prof. D. A. Indiana University, Bloomington, Ind. 1000 Atwater Avenue.

Rowe, Asst..Prof. J. E. Pennsylvania State College, State College, Pa. 415 Pugh Street.

Running, Jun. Prof. T. R. University of Michigan, Ann Arbor, Mich. 1019 Michigan Avenue.

Rusk, Prof. W. J. Grinnell College, Grinnell, Iowa. 1022 Park Street. Russell, Asso. Prof. W. P. Pomona College, Claremont, Cal.

RUtledge, George. University of Illinois, Urbana, Ill. 902 West Illinois Street. 
SAFrord, Asst. Prof. F. H. University of Pennsylvania, Philadelphia, Pa.

SANDERSON, Dr. MiLDRED L. Instructor, University of Wisconsin, Madison, Wis. 606 North Francis Street.

SAUREL, Prof. P. L. College of the City of New York, New York, N. Y.

SAYRe, Prof. H. A. University of Alabama, University, Ala.

SCARborough, Prof. J. H. Missouri State Normal School, Warrensburg, Mo.

*Schmiedel, Prof. Oscar. Bellevue College, Bellevue, Neb.

Schotrentrels, Miss I. M. 447 East 49th Street, Chicago, Ill.

Schwatt, Prof. I. J. University of Pennsylvania, Philadelphia, Pa.

Schweitzer, A. R. 452 Oakdale Avenue, Chicago, $\mathrm{Ill}$.

Scotw, Prof. Charlotte A. Bryn Mawr College, Bryn Mawr, Pa. 233 Roberts Road.

Searle, Rev. Dr. G. M. 660 California Street, San Francisco, Cal.

SEE, Prof. T. J. J. U. S. Naval Observatory, Mare Island, Cal.

Seely, Miss C. E. 523 West 121st Street, New York, N. Y.

SEguier, Rev. Dr. J. A. M. J. DE. 114 Rue du Bac., Paris, 7e, France.

SelLew, Prof. G. T. Knox College, Galesburg, Ill.

SharPe, Asst. Prof. F. R. Cornell University, Ithaca, N. Y. 213 Mitchell Street.

Shattuck, Prof. S. W. University of Illinois, Champaign, Ill.

SHAW, Asst. Prof. J. B. University of Illinois, Urbana, Ill. 901 California Avenue.

SheFrer, Dr. H. M. Instructor, University of Missouri, Columbia, Mo. 502 Turner Avenue.

Sheldon, Prof. E. W. University of Alberta, Edmonton South, Alberta, Canada.

Short, R. L. Principal, West Technical High School, Cleveland, Ohio.

Shumway, Asst. Prof. R. R. University of Minnesota, Minneapolis, Minn. 716 Twelfth Avenue, S. E.

SiCeloff, Asst. Prof. L. P. Columbia University, New York, N. Y.

Silverman, Dr. L. L. Instructor, Cornell University, Ithaca, N. Y.

Simpson, Asst. Prof. C. G. Pennsylvania State College, State College, $\mathrm{Pa}$.

Simpson, T. M. Instructor, University of Wisconsin, Madison, Wis. 308 Breeze Terrace.

Sinclatr, Asso. Prof. Mary E. Oberlin College, Oberlin, Ohio. 4 Randolph Road, Worcester, Mass.

*SIsam, Asst. Prof. C. H. University of Illinois, Urbana, Ill. 1304 South Orchard Street.

Skiles, Asso. Prof. W. V. Georgia School of Technology, Atlanta, Ga.

Skinner, Asso. Prof. E. B. University of Wisconsin, Madison, Wis. 210 Lathrop Street. 
Slaught, Prof. H. E. Univensity of Chicago, Chicago, Ill. $5548 \mathrm{Ken}$ wood Avenue.

SlePiAn, Dr. JosePH. Instructor, Cornell University, Ithaca, N. Y. 134 College Avenue.

*SLIChter, Prof. C. S. University of Wisconsin, Madison, Wis. 636 Frances Street.

Slobin, Asst. Prof. H. L. University of Minnesota, Minneapolis, Minn.

Slocum, Prof. S. E. University of Cincinnati, Cincinnati, Ohio.

SmaIL, Dr. L. L. Instructor, University of Washington, Seattle, Wash. 2121 East 55th Street.

SмIтH, Prof A. G. University of Iowa, Iowa City, Iowa.

Smith, Asso. Prof. A. W. Colgate University, Hamilton, N. Y.

*Smith, Asso. Prof. Clada E. Wellesley College, Wellesley, Mass. Shafer Hall.

*Sмiтh, Prof. D. E. Teachers College, Columbia University, New York, N. Y.

Smite, D. M. Instructor, Georgia School of Technology, Atlanta, Ga.

Smith, Asso. Prof. Edwin R. Pennsylvania State College, State College, Pa. North Campus.

Smith, Eugene R. Headmaster, The Park School, Auchentoroly Terrace, Baltimore, $\mathrm{Md}$.

Sмiтн, F. H. Eastern District High School, Brooklyn, N. Y. 414 West 120th Street, New York, N. Y.

Smith, Miss Gertrude. Instructor, Vassar College, Poughkeepsie, N. Y.

Sмiтн, Asst. Prof. I. W. North Dakota Agricultural College, Fargo, N. Dak. 1126 Thirteenth Street, North.

Smith, Prof. J. B. Hampden Sidney College, Hampden Sidney, Va.

Smitr, Prof. P. F. Sheffield Scientific School, Yale University, New Haven, Conn. 330 Willow Street.

Smith, Prof. SARAH E. Mount Holyoke College, South Hadley, Mass.

Sмiтн, Prof. W. B. Tulane University, New Orleans, La.

Sмiтн, Prof. W. M. University of Oregon, Eugene, Ore..

Sneling, Prof. C. M. University of Georgia, Athens, Ga. P. O. Box 283.

Snook, T. E. Architect and Engineer. 261 Broadway, New York, N. Y.

Snyder, Prof. M. B. Central High School, Philadelphia, Pa. 2402 North Broad Street.

SNyder, Prof. VIRGIL. Cornell University, Ithaca, N. Y. 214 University Avenue.

Sparrow, Adj. Prof. C. M. University of Virginia, Charlottesville, Va. SPITZER, George. Dairy Chemist, Purdue University Agricultural Experiment Station, Lafayette, Ind. Seventh and Waldron Streets, West Lafayette, Ind.

Stager, Prof. H. W. Fresno Junior College, Fresno, Cal. 265 Howard Street. 
Stamper, Dr. A. W. State Normal School, Chico, Cal.

*Stecker, Asso. Prof. H. F. Pennsylvania State College, State College, Pa. 306 Mills Street.

Steinmetz, Prof. C. P. Union College, Schenectady, N. Y. Chief Consulting Engineer, General Electric Company. Wendell Avenue.

Stephanos, Prof. Crparissos. University of Athens, Athens, Greece. 20 Rue Solon.

Stephens, Asso. Prof. R. P. University of Georgia, Athens, Ga.

Stetson, Dr. J. M. Lecturer, University of Alberta, Edmonton South, Alberta, Canada.

Stone, Prof. J. C. State Normal School, Montclair, N. J. 56 Macopin Avenue, Upper Montclair.

Stone, Prof. ORMOND. University of Virginia, Charlottesville, Va. Manassas, $\nabla a$.

Stone, Asst. Prof. R. B. Purdue University, Lafayette, Ind. 30ry Russell Street, West Lafayette.

STone, Asst. Prof. W. B. Rutgers College, Now Brunswick, N. J. "The Bayard."

Story, Prof. W. E. Clark University, Worcester, Mass.

Stouffer, Asst. Prof. E. B. University of Kansas, Lawrence, Kan. 308 West 16th Street.

Stromquist, Prof. C. E. University of Wyoming, Laramie, Wyo.

Strong, Dr. W. M. Associate Actuary, Mutual Life Insurance Company, New York, N. Y. 32 Nassau Street.

Study, Prof. Eduard. University of Bonn, Bonn, Germany. Argelanderstrasse 126.

Sullivan, Asst. Prof. C. T. McGill University, Montreal, Canada. Engineering Building.

Swartzel, Prof. K. D. Ohio State University, Columbus, Ohio. 1952 Iuka Avenue.

*Swift, Prof. EliJah. University of Vermont, Burlington, Vt. 433 South Willard Street.

*TABER, Prof. Henry. Clark University, Worcester, Mass.

*Tanner, Prof. J. H. Cornell University, Ithaca, N. Y. Cornell Heights.

TAPPan, Dr. AnNa H. Instructor, Iowa State College, Ames, Iowa. 241 Hyland Avenue.

TAYLoR, Dr. E. H. Eastern Illinois State Normal School, Charleston, IIl.

TAYLOR, Prof. J. M. Colgate University, Hamilton, N. Y.

Thomas, Prof. Evan. College of Engineering, University of Vermont, Burlington, Vt. 187 Loomis Street.

*Thompson, Prof. H. D. Princeton University, Princeton, N. J. 11 Morven Street. 
Thompson, J. S. Assistant Actuary, Mutual Life Insurance Company, New York, N. Y. 34 Nassan Street.

Thornburg, Prof. C. L. Lehigh University, South Bethlehem, Pa.

Touton, F. C. Principal, Central High School, St. Joseph, Mo. 13th and Olive Streets.

Townsend, Prof. E. J. University of Illinois, Champaign, Ill. 510 John Street.

Tracey, Dr. J. I. Instructor, Yale University, New Haven, Conn. 314 Norton Street.

TrIPP, Prof. M. O. Olivet College, Olivet, Mich.

Tyler, Prof. H. W. Massachusetts Institute of Technology, Boston, Mass.

Underhill, Asst. Prof. A. L. University of Minnesota, Minneapolis, Minn. 615 Sixth Street, S. $E$.

Upton, Asst. Prof. C. B. Teachers College, Columbia University, New York, N. Y.

URNeR, Asst. Prof. S. E. Miami University, Oxford, Ohio.

Van Amringe, Prof. J. H. Columbia University, New York, N. Y. 48 West 59th Street.

*Van Benschoten, Prof. Anna L. Wells College, Aurora, N. Y.

VAN DeR Heyden, A. F. Middlesbrough High School, Middlesbrough. England. 3 St. John's Terrace.

VAN DER VRIES, Prof. J. N. University of Kansas, Lawrence, Kan. 1644 New Hampshire Street.

Vandiver, H. S. 125 South Fifth Street, Philadelphia, Pa.

*Van Orstrand, C. E. Mathematical Physicist, U. S. Geological Survey, Washington, D. C. 1607 31st Street, N. W.

VAN VleCK, Prof. E. B. University of Wisconsin, Madison, Wis. 519 North Pinckney Street.

*Veblen, Prof. Oswald. Princeton University, Princeton, N. J.

VEDdER, J. N. Instructor, Union College, Schenectady, N. Y.

Vivian, Asso. Prof. R. H. Wellesley College, Wellesley, Mass. 3 Jefferson Hall, Trinity Court, Dartmouth Street, Boston, Mass.

Volterra, Prof. Vito. University of Rome, Rome, Italy. Via in Lucina, $1 \%$.

Waddeld, Miss M. E. G. 596 Spadina Avenue, Toronto, Canada.

WAHLIn, Dr. G. E. Associate, University of Illinois, Urbana, Ill. 903 Railroad Street.

WALDo, Prof. C. A. Washington University, St. Louis, Mo.

WALKer, Prof. B. M. Mississippi Agricultural and Mechanical College, Agricultural College, Miss.

WaLsh, C. B. Ethical Culture School, New York, N. Y. Hotel Monticello, 35 West 64th Street. 
Washburne, A. C. Actuary, Berkshire Life Insurance Company, Pittsfield, Mass.

WAtKeys, Asst. Prof. C. W. University of Rochester, Rochester, N. Y. WeBb, H. E. Central Commercial and Manual Training High School, Newark, N. J. 12 Irving Place, Summit, N. J.

Webster, Prof. A. G. Clark University, Worcester, Mass. 66 West Street.

Wedderburn, Asst. Prof. J. H. M. Princeton University, Princeton, N. J. Nassau Club.

WeEks, Miss E. A. University of Missouri, Columbia, Mo. 817 Virginia Avenue.

WEEKS, R. W. Vice-President and Chief Actuary, New York Life Insurance Company, New York, N. Y. 346 Broadway.

WeLd, Prof. L. G. Pullman School of Manual Training, Pullman, Chicago, IIl.

WeLls, Asso. Prof. Mary E. Oberlin College, Oberlin, Ohio. 20 North Pleasant Street.

Wells, Prof. Webster. Massachusetts Institute of Technology, Boston, Mass. Hotel Beaconsfield, Brookline, Mass.

Wentworth, George. 187 Winthrop Road, Brookline, Mass.

West, Asst. Prof. C. J. Ohio State University, Columbus, Ohio. 105 DeWitt Place, Ithaca, N. Y.

WeSTER, C. W. Instructor, University of Iowa, Iowa City, Iowa. 225 Fairchild Street.

*Western, Dr. A. E. 27 Pembridge Square, London, W., England.

Westrall, Asso. Prof. W. D. A. University of Missouri, Columbia, Mo. 309 Hicks Avenue.

Westudnd, Prof. JАсов. Purdue University, Lafayette, Ind. 628 South Street.

Wetzes, R. A. Instructor, College of the City of New York, New York, N. Y. 505 West $142 d$ Street.

WHITE, Prof. H. S. Vassar College, Poughkeepsie, N. Y.

White, Asso. Prof. Marion B. Michigan State Normal College, Ypsilanti, Mich. 126 North Washington Street.

WHITFORD, Asst. Prof. E. E. College of the City of New York, New York, N. Y. 180 Claremont Avenue.

Whitney, A. W. General Manager, Workmen's Compensation Service Bureau, New York, N. Y. 18 East 41 st Street.

*WhitTaker, Prof. E. T. University of Edinburgh, Edinburgh, Scotland. 35 George Square.

*Whittemore, J. K. Pomfret School, Pomfret Center, Conn.

WILCZynski, Prof. E. J. University of Chicago, Chicago, Ill. 5332 Kimbark Avenue.

Wilex, Prof. F. B. Denison University, Granville, Ohio,

WiLlard, Prof. J. M. Pennsylvania State College, State College, Pa. 
Williams, Prof. C. B. Kalamazoo College, Kalamazoo, Mich. 214 Stuart Avenue.

Williams, Miss E. C. Miss Spence's School, 30 West 55th Street, New York, N. Y.

Winliams, Prof. F. B. Clark University, Worcester, Mass.

Willifams, Prof. J. E. Virginia Polytechnic Institute, Blacksburg, Va.

Willitams, Asst. Prof. K. P. Indiana University, Bloomington, Ind. 405 North Indiana Avenue.

Williams, Prof. W. H. State Normal School, Platteville, Wis.

Willson, Prof. F. N. Princeton University, Princeton, N. J. P. $O$. Box 63.

Wirson, Asso. Prof. A. H. Haverford College, Haverford, Pa.

Wruson, Asso. Prof. D. T. Case School of Applied Science, Cleveland, Ohio.

WILson, Prof. E. B. Massachusetts Institute of Technology, Boston, Mass.

WiLson, Prof. N. R. University of Manitoba, Winnipeg, Canada. 75 Sherbrooke Street.

WILson, Asst. Prof. R. E. Northwestern University, Evanston, Ill.

Wirson, Asst. Prof. W. A. Yale University, New Haven, Conn. 705 Elm Street.

Winger, Asst. Prof. R. M. University of Oregon, Eugene, Ore.

WolfF, Asst. Prof. H. C. University of Wisconsin, Madison, Wis. 6 South Prospect Avenue.

Wood, Prof. RutH G. Smith College, Northampton, Mass. 249 Crescent Street.

Woods, Prof. F. S. Massachusetts Institute of Technology, Boston, Mass.

*Woodward, Pres. R. S. Carnegie Institution, Washington, D. C.

Worthington, Dr. Euphemia R. Instructor, Wellesley College, Wellesley, Mass. $10 \mathrm{Waban}$ Street.

WRIGHT, W. C. Actuary. 204 Forest Street, Medford, Mass.

YANNEY, Prof. B. F. College of Wooster, Wooster, Ohio.

Yeaton, C. H. University of Chicago, Chicago, mll.

Young, Prof. A. E. Miami University, Oxford, Ohio.

Young, Asso. Prof. J. W. A. University of Chicago, Chicago, Ill. 5422 Blackstone Avenue.

Young, Prof. J. W. Dartmouth College, Hanover, N. H.

Young, Dr. MabeL M. Instructor, Wellesley College, Wellesley, Mass. 6 Norfolk Terrace.

YoweLL, Dr. E. I. First Astronomer, Cincinnati Observatory, Cincinnati, Ohio. Griest and Corbett Avenues. 
Zehring, Asst. Prof. W. A. Purdue University, Lafayette, Ind. 303 Russell Street, West Lafayette, Ind.

Zervos, Prof. Panaiotis. University of Athens, Athens, Greece. Rue Filis, $8 \%$.

Zrwet, Prof. Alexander. University of Michigan, Ann Arbor, Mich. 644 South Ingalls Street.

Number of members, January $1,1914 \ldots \ldots \ldots \ldots \ldots \ldots \ldots \ldots 703$

Number of members, January $1,1915 \ldots \ldots \ldots \ldots \ldots \ldots \ldots \ldots . \ldots . \ldots . \ldots$

Members admitted during the year $1914 \ldots \ldots \ldots \ldots \ldots \ldots \ldots . \ldots . \ldots . \ldots$

Members withdrawing during the year $1914 \ldots \ldots \ldots \ldots \ldots \ldots, 32$

Number of life members.......................... 69 